Annals of Pure and Applied Mathematics

Vol. 16, No. 2, 2018, 487-499

ISSN: 2279-087X (P), 2279-0888(online)

Published on 31 March 2018

www.researchmathsci.org

DOI: http://dx.doi.org/10.22457/apam.v16n2a27

Annals of

Pure and Applied

Mathematics

\title{
An EPQ model for Breakable Item under Imperfect Production Process
}

\author{
A.Kundu ${ }^{1}$, P.Guchhait ${ }^{2}$, G.Panigrahi ${ }^{1}$ and M.Maiti $^{2}$ \\ ${ }^{1}$ Department of Mathematics, National Institute of Technology \\ Mahatma Gandhi Avenue, Durgapur-713209, West Bengal, India. \\ Email:kundua2012@gmail.com, panigrahi_goutam@ rediffmail.com \\ ${ }^{2}$ Department of Applied Mathematics, Vidyasagar University \\ Midnapore, West Bengal, India-721102 \\ E-mail: parthaguchhait@ gmail.com; mmaiti2005@yahoo.co.in \\ Received 28 February 2018; accepted 29 March 2018
}

\begin{abstract}
In the present investigation, an economic production quantity (EPQ) model for breakable item has been developed with time-sensitive demand and breakability rate. Production process is not perfect and as a result, produces some imperfect quality units. Breakability rate of the item starts above a certain stock level and increases with time as well as stock level. Planning horizon is assumed to be stochastic in nature and follows normal distribution with a known mean and standard deviation. Production cost per unit depends on the production rate and is derived from the particular production function under which it is being produced. As the present competitive market situation is full of uncertainty, another model is developed with the consideration of inventory parameters as fuzzy in nature and obtained results are presented. Numerical experiments are performed to illustrate the model.
\end{abstract}

Keywords: EPQ model, Stock and time dependent breakability, Time-sensitive demand, Random planning horizon.

AMS Mathematics Subject Classification (2010): 90B05

\section{Introduction}

Now-a-days, an economic production quantity (EPQ) model is one of the most essential methodology and widely applied in the industrial belt or any production sector. The earlier EPQ models are mostly used to solve the problem of the optimal lot size or the production runtime to maximize the profit. One of the basic assumptions of the classical EPQ model is that all the items produced are of perfect quality. However, in the realistic situations, production process is not perfect throughout the process runtime. Thus, the assumption of perfect quality production process is not fit for the industrial applications. In 1980, Shih [1] investigated first the effect of defective products on the inventory cost and also on the production system. After that lot of research works have been published 


\section{A.Kundu, P.Guchhait G.Panigrahi and M.Maiti}

on EPQ models with imperfect quality items along with different types of assumptions (Bakker et al. [2], Hso and Hso [3], Kundu et al. [4], Ghosh [5], etc). In addition, the earlier models also implicitly assume that the items produced are of perfect quality. Naturally, it is not happened always and thus, a high-level production quality can only be achieved with substantial investment in improving the reliability of the production process. Beek and Puttin [6] have addressed the issue of flexibility improvement production. Issue of process reliability, quality improvement, and set-up time reduction have been discussed by Porteus [7], Rosenblatt and Lee [8], etc. They assumed that the perfect quality units are produced in the in-control state (i.e., beginning of the production process) and the imperfect quality products are produced in out-of-control state.

Sometimes, manufacturer of the breakable items like glass, items made of mud, electronic plastic toys, etc., faces a conflicting situation in their production run time. To decrease the production cost and set-up cost, they are tempted to go for a large number of production and at the same time invites more damage to his units. Some research papers has already been published in this direction (Maragatham and Gnanvel [9], Guchhait et al. [10], Mandal and Maiti [11]). On the other hand, it is observed that pressure of one unit onto the other for a long time may increase the breakability rate. So far, in the literature, there are only few number of investigations are presented with this types of realistic assumption.

Most of the production inventory models have been formulated with infinite time horizon assuming that the demand of the item remains same for ever (Maiti and Maiti [12], Saha and Cardenas-Barron [13], Arif [14], etc). According to this assumption, product specification remains unchanged for ever, but in reality unprecedental development of technology has led to rapid change in product specifications (Gurnani, [15]) with new features. Few investigation have already been published incorporating this type of assumption (Moon and Yun [16], Pal et al. [17], Kundu et al. [4]). On the other hand, items in the market, such as Tab, i-pad, hi-fi equipment, breakable items such electronic plastic toys, ceramic, etc. are highly demandable, but normally exist in the market for a finite time and obviously these types of demands depend on time. Various types of investigations have already been made by several authors in this direction (Lee and Hsu [18], Guchhait et al. [10], Kundu et al. [19], Mariappan et al. [20]). Again, lifetime of these types of products is finite and normally it is imprecise (stochastic, fuzzy, etc.) in nature. In the present investigation demand of the item is considered as time dependent and planning horizon is assumed to be random in nature.

Cost of production per unit of an item in any manufacturing system depends upon several production related factors such as raw materials, technical knowledge, production rate, etc. Khouja [21] first developed an EPL model under volume flexibility, where unit production cost depends upon the raw materials used, and labour force engaged and tool wear and tear cost incurred. Production rate is also an important factor in any production sector. Increase of production rate decreases the unit cost which in turn increases demand. Again, increase of production rate increases holding cost which decreases profit. Hence, the manufacturers face the above mentioned contradiction and to overcome this situation they try to make an optimal decision for maximum profit. 
An EPQ model for Breakable Item under Imperfect Production Process

Khouja and Mehrez [22] presented a classical inventory model and showed that the optimal production rate is smaller than the production rate which minimizes the unit production cost.

In the present investigation, an imperfect EPQ model for breakable item has been developed in a random planning horizon. Breakability rate of the item increases not only the stock label, but also increases with time. Produced defective units are sold at a reduced price. As the model is developed for the fashionable/decorating items, demand is considered as time-sensitive. Production cost per unit depends on production rate, raw material cost, labor, tool wear and tear costs. A general equation, proposed by Cheng [23], is used to develop the model and also the relationship between set-up cost and process reliability and flexibility. As today's competitive volatile business transaction is full of uncertainty, inventory/production-inventory policy with fuzzy coefficients is a well-established phenomenon in recent years. After introduction of fuzzy set (Zadeh [24]), it has been well developed and applied widely in different areas of science and technology including inventory control problems (Maiti [25], Guchhait et al. [26, 27, 28]). Another model is developed considering some inventory parameters as a fuzzy number. The present model is solved following GRG technique (Using LINGO software). Numerical examples are used to illustrate the model.

\section{Notations and assumptions for the proposed model}

Notations:

$P$

$c_{h}$

$c_{p}$

$r_{m}$

$s_{p}$

$s_{p r}$

$\xi_{0}$

$t_{0}$

$t_{1}$

$t_{0}^{\prime}$

$T$

$N$

$I_{1}$

$I(t)$
Explanations

Production Rate

Holding cost per unit in \$

Production cost per unit in $\$$

Raw material cost in $\$$

Selling price per unit in $\$$

Selling price per unit for broken unit in \$

Set-up cost per cycle in $\$$

Time when inventory level reaches $\mathrm{I}_{0}$ during production

Time when inventory level reaches $I_{1}$ during production

Time when inventory level reaches $\mathrm{I}_{0}$ after production

Cycle length in days

Number of full cycle during the planning horizon

Highest inventory level

Inventory level at any time $\mathrm{t}$

Assumptions:

(i) Inventory system involves only one breakable item.

(ii) Breakability of the item $B(I, t)$ is stock and time dependent and is of

the form: $B(I, t)=\left\{\begin{array}{cc}\gamma\left[I(t)-I_{0} e^{-\psi\left(t-t_{0}\right)}\right] ; & t_{0} \leq t \leq t_{0}^{\prime} \\ 0 & \text { elsewhere }\end{array}\right.$

where $\gamma$ and $\psi$ are constant

(iii) Demand of the item $D$ is considered as time sensitive, i.e., $D=D(t)$ 


\section{A.Kundu, P.Guchhait G.Panigrahi and M.Maiti}

(iv) Production cost $c_{p}$ is a known function of raw material cost $r_{m}$, the production rate $P$ and is of the form: $c_{p}=r_{m}+\frac{L}{P^{\sigma_{1}}}+T_{W} P^{\sigma_{2}}$ where $L$ is labor charges and $T_{W}$ is the tool wear and tear cost. $\sigma_{1}$ and $\sigma_{2}$ are two positive constants.

(v) $\quad \xi_{0}$ is set-up cost per cycle in $\$$

(vi) Total cost of interest and depreciation per production cycle is inversely related to set-up cost $\left(\xi_{0}\right)$ and directly relatedto process reliability $(r)$ according to the following relation (Cheng 1989):

$$
F\left(\xi_{0}, r\right)=\chi \xi_{0}{ }^{-\beta_{1}} r^{\beta_{2}}
$$

where $\chi, \beta_{1}, \beta_{2}$ are positive constants.

(vii) $\widehat{H}$ is planning horizon which is random in nature.

(viii) $\quad N$ Number of full cycle are completed during the planning horizon.

(ix) Selling price $s_{p}=m c_{p}$, where $m$ is the mark-up of the production cost and $m>1$.

(x) Broken and defective units are sold at a reduced price $s_{p r}=m_{r} c_{p}$, where $m_{r}$ is the mark-up of the production cost and $m_{r}<1$.

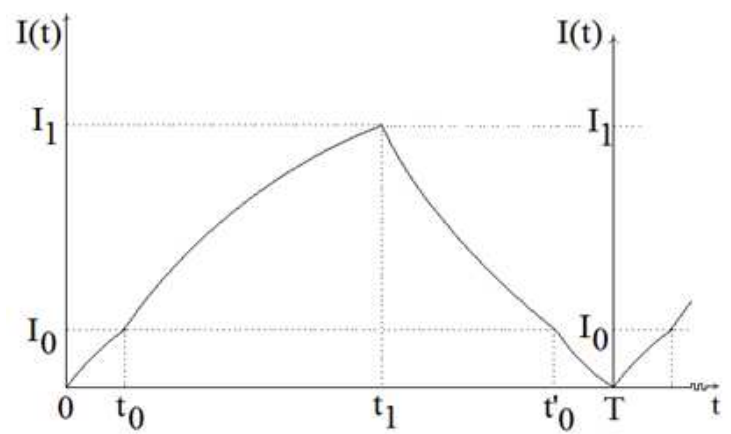

Figure 1: Inventory level with time

\section{Mathematical formulation}

In the development of the model, it is assumed that the manufacturer produces the fresh units at a rater $P$. So the produced defective units are $(1-r) P$ and these defective units are sold at a reduced price. Production process runs upto the time $t_{l}$ and after that customer's demand is met using the stored units. At the end of each cycle inventory level reaches zero and then production for next cycle starts. According to the above assumptions, the change of inventory level at any time $t$, are given by

$$
\frac{d I}{d t}= \begin{cases}r P-D(t) ; & 0 \leq t \leq t_{0} \\ r P-D(t)-B(I, t) ; & t_{0} \leq t \leq t_{1} \\ -D(t)-B(I, t) ; & t_{1} \leq t \leq t_{0}^{\prime} \\ -D(t) ; & t_{0}^{\prime} \leq t \leq T\end{cases}
$$

With boundary conditions $I(0)=0, I\left(t_{0}\right)=I_{0}, I\left(t_{1}\right)=I_{1}, I\left(t_{0}^{\prime}\right)=I_{0}, I(T)=0$, 
An EPQ model for Breakable Item under Imperfect Production Process

Here, we considered that the demand is increasing at a decreasing rate and the function is of the form $D(t)=a_{1}-b_{1} e^{-c_{1} t}$ where $a_{1}, b_{1}, c_{1}$ are positive constants.

Now, solving the above differential equation (1) and using the boundary conditions, we get

$$
I(t)=\left\{\begin{array}{c}
\left(r P-a_{1}\right) t+b_{1}\left(1-e^{-c_{1} t}\right) / c_{1} \\
K_{44} e^{-\gamma\left(t-t_{0}\right)}+K_{33} e^{-\psi t}+K_{22} e^{-c_{1} t}+K_{11} \\
K_{55} e^{-\gamma\left(t-t_{1}\right)}+K_{88} e^{-\psi t}+K_{77} e^{-c_{1} t}+K_{66} \\
\left(I_{0}-\frac{b_{1} e^{-c_{1} t}}{c_{1}}\right)-a_{1}\left(t-t_{0}^{\prime}\right)+b_{1} e^{-c_{1} t} / c_{1}
\end{array}\right.
$$

where $K_{11}=\left(r P-a_{1}\right) / \gamma, K_{22}=b_{1} /\left(\gamma-c_{1}\right), K_{33}=\gamma I_{0} e^{\psi t_{0}} /(\gamma-\psi), K_{44}=I_{0}-$ $K_{11}-K_{22} e^{-c_{1} t_{0}}-K_{33} e^{\psi t_{0}}, \quad K_{55}=I_{1}+\frac{a_{1}}{\gamma}-\frac{b_{1} e^{-c_{1} t_{1}}}{\gamma-c_{1}}-\gamma I_{0} e^{\left(t_{0}-t_{1}\right)} /(\gamma-\psi), \quad K_{66}=$ $-\frac{a_{1}}{\gamma}, K_{77}=\frac{b_{1}}{\gamma-c_{1}}, K_{88}=\gamma I_{0} e^{\psi t_{0}} /(\gamma-\psi)$

Now using boundary conditions we get,

$I_{0}=\left(r P-a_{1}\right) t_{0}+b_{1}\left(1-e^{-c_{1} t_{0}}\right) / c_{1}$

$I_{1}=K_{44} e^{-\gamma\left(t_{1}-t_{0}\right)}+K_{33} e^{-\psi t_{1}}+K_{22} e^{-c_{1} t_{1}}+K_{11}$

$I_{0}=K_{55} e^{-\gamma\left(t_{0}^{\prime}-t_{1}\right)}+K_{88} e^{-\psi t_{0}^{\prime}}+K_{77} e^{-c_{1} t_{0}^{\prime}}+K_{66}$

$I_{0}+b_{1}\left(e^{-c_{1} T}-e^{-c_{1} t_{0}}\right) / c_{1}-a_{1}\left(T-t_{0}^{\prime}\right)=0$

From these equations we can get the values of $t_{0}, t_{1}, t_{0}^{\prime}$ and $T$

\section{Inventory cost calculation:}

Total holding cost from the cycle is $c_{h} C_{H}$ and $C_{H}$ is given by

$$
\begin{aligned}
C_{H} & =\int_{0}^{T} I(t) d t=\int_{0}^{t_{0}} I(t) d t+\int_{t_{0}}^{t_{1}} I(t) d t+\int_{t_{1}}^{t_{0}^{\prime}} I(t) d t+\int_{t_{0}^{\prime}}^{T} I(t) d t \\
& =C H_{1}+C H_{2}+C H_{3}+C H_{4} \text { (say) }
\end{aligned}
$$

where, $C H_{1}=\frac{\left(r P-a_{1}\right) t_{0}^{2}}{2}+\frac{b_{1} t_{0}}{c_{1}}+\frac{b_{1}\left(e^{-c_{1} t_{0}}-1\right)}{c_{1}^{2}}$,

$$
\begin{aligned}
& C H_{2}=\frac{K_{44}\left(1-e^{-\gamma\left(t_{1}-t_{0}\right)}\right)}{\gamma}+\frac{K_{33}\left(e^{\psi t_{0}}-e^{\psi t_{1}}\right)}{\psi}+\frac{K_{22}\left(e^{-c_{1} t_{0}}-e^{-c_{1} t_{1}}\right)}{c_{1}}+K_{11}\left(t_{1}\right. \\
& \left.-t_{0}\right) \mathrm{CH}_{3} \\
& =\frac{K_{55}\left(1-e^{-\gamma\left(t_{0}^{\prime}-t_{1}\right)}\right)}{\gamma}+\frac{K_{88}\left(e^{\psi t_{1}}-e^{\psi t_{0}^{\prime}}\right)}{\psi}+\frac{K_{77}\left(e^{-c_{1} t_{1}}-e^{-c_{1} t_{0}^{\prime}}\right)}{c_{1}} \\
& +K_{66}\left(t_{0}^{\prime}-t_{1}\right) \mathrm{CH}_{4} \\
& =\left(I_{0}-\frac{b_{1} e^{-c_{1} t_{0}}}{c_{1}}\right)\left(T-t_{0}^{\prime}\right)-\frac{a_{1}\left(T-t_{0}^{\prime}\right)^{2}}{2}-b_{1}\left(e^{-c_{1} T}-e^{-c_{1} t_{0}^{\prime}}\right) / c_{1}^{2}
\end{aligned}
$$

Total sale revenue from one cycle is $s_{p} R_{S}$, where

$R_{S}=\int_{0}^{T} D(t) d t=a_{1} T+b_{1}\left(e^{-c_{1} T}-1\right) / c_{1}$

The salvage value iss ${ }_{p r} R_{V}$ and is given by 
A.Kundu, P.Guchhait G.Panigrahi and M.Maiti

$R_{V}=\int_{t_{0}}^{t_{0}^{\prime}} B(I, t) d t=\gamma\left(C H_{2}+C H_{3}\right)+\gamma I_{0}\left(e^{-\psi\left(t_{0}^{\prime}-t_{0}\right)}-1\right) / \psi$

Where $\mathrm{CH}_{2}$ and $\mathrm{CH}_{3}$ are given above.

Hence, during the whole planning horizon, total profit $\left(P_{T}\right)$ can be written as $N P_{T}$ where $P_{T}$ is given by the following equation

$P_{T}=s_{p} R_{S}+s_{p r}\left[R_{V}+(1-r) P t_{1}\right]-c_{p} P t_{1}-c_{h} C_{H}-\xi_{0}-F\left(\xi_{0}, r\right)$

\subsection{Optimization in different environments}

Depending upon the nature of the parameters of optimization problems, various types of optimization problems are faced by the researchers. To the authors' best knowledge, till now, none has solved non-deterministic problems directly except crisp optimization problems. Different approaches are used to transform these problems into crisp environment. The approaches used in this paper are summarized below.

\subsubsection{Crisp optimization problem}

A crisp non-linear problem in crisp environment may be defined as follows:

Determine $x_{1}, x_{2}, \ldots x_{n}$, to

Maximize $\left.f\left(x_{1}, x_{2}, \ldots x_{n}, a_{1}, a_{2}, \ldots a_{n}\right)\right\}$

where $x_{1}, x_{2}, \ldots x_{n}$ are crisp decision variables and $a_{1}, a_{2}, \ldots a_{n}$ are crisp parameters.

\subsubsection{Fuzzy optimization problem}

When the parameters, such as $a_{1}, a_{2}, \ldots a_{n}$ are fuzzy (triangular type) in nature (i.e., $\tilde{a}_{1}, \tilde{a}_{2}, \ldots \tilde{a}_{n}$ are fuzzy parameters), the above problem (11) reduces to a fuzzy optimization problem as

$$
\left.\begin{array}{c}
\text { Determine } x_{1}, x_{2}, \ldots x_{n}, \text { to } \\
\text { Maximize } \tilde{f}\left(x_{1}, x_{2}, \ldots x_{n}, \tilde{a}_{1}, \tilde{a}_{2}, \ldots \tilde{a}_{n}\right)
\end{array}\right\}
$$

Solution Technique: As the optimization of fuzzy objective is not well defined, different authors take the different crisp equivalent of this objective to determine the approximate solution for the decision maker (DM). Here, Graded Mean Integration Representation (GMIR) method of the fuzzy objective is taken as an equivalent crisp objective and hence the problem (12) reduced to

$\left.\begin{array}{c}\text { Determine } x_{1}, x_{2}, \ldots x_{n}, \text { to } \\ \text { Maximize GMIR of } \tilde{f}\left(x_{1}, x_{2}, \ldots x_{n}, \tilde{a}_{1}, \tilde{a}_{2}, \ldots \tilde{a}_{n}\right)\end{array}\right\}$

Crisp Model (CM): As the planning horizon is taken as random in nature, so the problem is to

Determine the value of $P, r$ and $\xi_{0}$ to

$$
\begin{gathered}
\text { Maximize } N P_{T} \\
\text { subject to } N T-\widehat{H} \leq 0
\end{gathered}
$$

If $p_{r}$ be the probability of realizing the constraint of (14), then the problem (14) reduces to Determine the value of $P, r$ and $\xi_{0}$ to

$$
\left.\begin{array}{c}
\text { Maximize } N P_{T} \\
\text { subject to } P(N T-\widehat{H} \leq 0) \geq p_{r}
\end{array}\right\}
$$


An EPQ model for Breakable Item under Imperfect Production Process

Using Charns constraint programming approach (cf. Appendix-A) the problem reduces to Determine the value of $P, r$ and $\xi_{0}$ to

$$
\left.\begin{array}{c}
\text { Maximize } N P_{T} \\
\text { ject to } N T \leq \bar{H}-\epsilon \sigma_{H}
\end{array}\right\}
$$

where $\bar{H}$ and $\sigma_{H}$ are mean and standard deviation (normal distribution) of the random variable $\widehat{H}$.

Fuzzy Model (FM): Here, it is assumed that some inventory costs of the item are triangular fuzzy number (TFN) (Zadeh, [24]), such as mark-up of the selling price per unit $\widetilde{m}=\left(m_{1}, m_{2}, m_{3}\right)$ (hence $\tilde{s}_{p}=\left(s_{p 1}, s_{p 2}, s_{p 3}\right)$, mark-up of the selling price of deteriorated units $\tilde{m}_{r}=\left(m_{r 1}, m_{r 2}, m_{r 3}\right)$, and the holding cost $\tilde{c}_{h}=\left(c_{h 1}, c_{h 2}, c_{h 3}\right)$. Then, the expression of the total fuzzy profit $P_{T F}=\left(P_{T F 1}, P_{T F 2}, P_{T F 3}\right)$, where

$$
\begin{aligned}
& P_{T F 1}=s_{p 1} R_{S}+s_{p r 1}\left[R_{V}+(1-r) P t_{1}\right]-c_{p} P t_{1}-c_{h 3} C_{H}-\xi_{0}-F\left(\xi_{0}, r\right) \\
& P_{T F 2}=s_{p 2} R_{S}+s_{p r 2}\left[R_{V}+(1-r) P t_{1}\right]-c_{p} P t_{1}-c_{h 2} C_{H}-\xi_{0}-F\left(\xi_{0}, r\right) \\
& P_{T F 3}=s_{p 3} R_{S}+s_{p r 3}\left[R_{V}+(1-r) P t_{1}\right]-c_{p} P t_{1}-c_{h 1} C_{H}-\xi_{0}-F\left(\xi_{0}, r\right)
\end{aligned}
$$

Following Graded Mean Integration Representation method of fuzzy number (cf. Appendix-B), defuzzifying $\tilde{P}_{T F}$ we get

$P_{T F}=\frac{1}{3}\left[k\left(P_{T F 1}+2 P_{T F 2}\right)+(1-k)\left(2 P_{T F 2}+P_{T F 3}\right)\right]$

Now the problem reduces to

Determine the value of $P, r$ and $\xi_{0}$ to

$$
\left.\begin{array}{c}
\text { Maximize } N P_{T F} \\
\text { subject to } N T-\widehat{H} \leq 0
\end{array}\right\}
$$

Using Charns constraint programming approach (cf. Appendix-A) in the same way as in crisp model, the problem reduces to

Determine the value of $P, r$ and $\xi_{0}$ to

$$
\left.\begin{array}{c}
\text { Maximize } N P_{T F} \\
\text { subject to } N T \leq \bar{H}-\epsilon \sigma_{H}
\end{array}\right\}
$$

where $P_{T F}$ is given by (17).

\section{Numerical illustration}

\subsection{Crisp model (CM)}

The following parametric values are used to illustrate the crisp model:

$a_{1}=80 ; b_{1}=30 ; c_{1}=5 ; r_{m}=1.3 \$ ; L=15 ; T_{W}=0.11 ; \sigma_{1}=2.1 ; \sigma_{2}=0.15 ; \chi=$ $160 ; \beta_{1}=0.5 ; \beta_{2}=4 ; \gamma=0.03 ; I_{0}=31 ; m=2.2 ; m_{r}=0.8 ; \bar{H}=180 ; \sigma_{H}=$ $1.5 ; c_{h}=0.2$ For stochastic constraint degree of probability $p_{r}$ is taken as 0.95 , i.e., $\epsilon=1.645$

With these parametric values, results are obtained and presented in the following table, i.e., Table-1.

Table 1: Optimum results of crisp model

\begin{tabular}{|c|c|c|c|c|c|c|}
\hline$P$ & $r$ & $\xi_{0}$ & $N$ & $t_{1}$ & $T$ & $P_{T}$ (Profit) \\
\hline 84.47 & 0.9613 & 18.56 & 5 & 34.27 & 35.51 & 24009.72 \\
\hline
\end{tabular}




\section{A.Kundu, P.Guchhait G.Panigrahi and M.Maiti}

For these assumed parametric values, results are also obtained due to the different production rates and presented in Table- 2 .

Table 2: Optimum results for different values of $P$

\begin{tabular}{|c|c|c|c|c|c|c|}
\hline$P$ & $r$ & $\xi_{0}$ & $N$ & $t_{1}$ & $T$ & $P_{T}$ (Profit) \\
\hline 83 & 0.9687 & 18.65 & 4 & 43.15 & 44.38 & 23967.08 \\
\hline 84 & 0.9658 & 18.56 & 4 & 43.15 & 44.38 & 23992.95 \\
\hline 85 & 0.9576 & 18.53 & 6 & 28.36 & 29.59 & 23995.75 \\
\hline 86 & 0.9481 & 18.28 & 7 & 24.13 & 25.36 & 23861.51 \\
\hline 87 & 0.9467 & 18.14 & 9 & 18.49 & 19.73 & 23818.88 \\
\hline
\end{tabular}

\subsection{Fuzzy model (FM)}

Here, another model is considered with the assumption that some parameters like $m, m_{r}$ and $c_{h}$,etc. are fuzzy in nature. This model is illustrated with the above example whose parametric values are same as in crisp model except the fuzzy variables and which are presented below:

$\widetilde{m}=\left(m_{1}, m_{2}, m_{3}\right)=(2.15,2.20,2.25), \tilde{m}_{r}=\left(m_{r 1}, m_{r 2}, m_{r 3}\right)=(0.70,0.75,0.82), \tilde{c}_{h}=$ $\left(c_{h 1}, c_{h 2}, c_{h 3}\right)=(0.18,0.20,0.23)$. Other parametric values are same as in crisp model. With these set of input data, optimum results of the fuzzy model are obtained and presented in the Table- 3 and -4 .

Table 3: Optimum results of fuzzy model

\begin{tabular}{|c|c|c|c|c|c|c|}
\hline$P$ & $r$ & $\xi_{0}$ & $N$ & $t_{1}$ & $T$ & $P_{T}$ (Profit) \\
\hline 83.18 & 0.9503 & 18.51 & 4 & 43.34 & 44.38 & 23861.13 \\
\hline
\end{tabular}

Table 4: Optimum results of FM for different production rate $(P)$

\begin{tabular}{|c|c|c|c|c|c|c|}
\hline$P$ & $r$ & $\xi_{0}$ & $N$ & $t_{1}$ & $T$ & $P_{T}$ (Profit) \\
\hline 82 & 0.9597 & 18.61 & 2 & 87.55 & 88.78 & 23799.22 \\
\hline 83 & 0.9536 & 18.54 & 3 & 57.88 & 59.39 & 23829.49 \\
\hline 84 & 0.9454 & 18.31 & 4 & 43.34 & 44.38 & 23843.55 \\
\hline 85 & 0.9425 & 18.25 & 6 & 28.35 & 29.58 & 23811.93 \\
\hline 86 & 0.9381 & 17.98 & 6 & 28.36 & 29.59 & 23782.26 \\
\hline
\end{tabular}

\section{Discussion}

Results of the crisp model are presented in Table-1, when production rate, reliability of the production process and set-up cost are decision variables. For different production rates, results are obtained and presented in Table-2. It is observed from Table-2 that initially profit increases with production rate, but after a certain level, profit decreases with increase of the production rate. Reliability of the production process gradually decreases with the increase of production rate and it agree with reality. As the reliability of the production process decreases, total cost of interest and depreciation per production cycle $\left(F\left(\xi_{0}, r\right)\right)$ decreases. Which take part in increase of the total profit ? On the other hand, decrease of process reliability indicates more defective units are produced during 
An EPQ model for Breakable Item under Imperfect Production Process

the production run time, which in turn decreases the profit. It can be concluded that, initially as production rate increases, 'gain' due to decrease of the depreciation cost $\left(F\left(\xi_{0}, r\right)\right)$ dominates 'loss' due to increase of defective produced units and obviously resultant profit increases. After crossing a certain production rate, 'gain' is dominated by the 'loss' and for which resultant profit decreases.

Again, to avoid high holding cost, cycle length $(T)$ decreases (with the increasing production rate), which in turn increases total number of cycles $(N)$ in the whole planning horizon. On the other hand, as $N$ increase, set-up cost and machine depreciation cost also increase and as a result, total profit from the planning horizon decreases. With the initial production rate, resultant effect of increasing profit dominates this fact, but after a certain production rate this is more effective for the fact of decreasing profit. This type of observation is reflected from both the tables, i.e., Table-2 and Table-4. From Table-3, it can be said that the nature of the obtained result is similar to the crisp one. More production rate produces large number of units as well as defective ones, together with more set-up and holding costs. Hence, in any production sector, maximum profit depends on production rate for the imperfect production process. Again, it is merely impossible for the manufacturer to maintain the optimal production rate. So, it is difficult for the manager to take decision in favour of any sector. In that case according to firm's facility a production rate near to optimal one is followed as a compromise decision.

\section{Conclusion}

In the present investigation, an imperfect EPQ model for breakable item is developed with stock and time dependent breakability rate. Here, planning horizon is assumed to be finite and stochastic in nature. The model is developed especially for seasonal products. Demand of the item increases with time at a decreasing rate. Total cost of interest and depreciation per production cycle, i.e. $F\left(\xi_{0}, r\right)$ is considered as a function of the setup(decision)variable $\xi_{0}$. Again, present competitive market situation is full of uncertainty. To deal with the uncertain inventory costs, parameters may also be considered as probabilistic in nature. In fact, estimation of probabilistic parameters are made on the basis of sufficient amount of past data. On the other hand, when past data is insufficient, one has to depend on fuzzy representation. As the estimation of fuzzy parameters can be made using expert's opinion, hence, for the present problem fuzzy model is adopted. Some interesting observations are made among which most important are (i) Maximum profit mostly depends on production rate, and (ii) High reliability of the production process may not give more profit always in an imperfect production system. Here, the formulation and solutions are quite general and hence the present models can be extended as a multi item inventory model to include more constraints such as available space, available budget, etc. The present investigation may include different types of demand functions- stock dependent, promotional effort dependent, etc. This model can also be extended to include different types of imprecise inventory costs such as, rough, fuzzyrough, etc. 


\section{A.Kundu, P.Guchhait G.Panigrahi and M.Maiti}

\section{REFERENCES}

1. W.Shih, Optimal inventory policies when stockouts result from defective products, International Journal of Production Research, 18 (1980) 677-686.

2. M.Bakker, J.Riezebos, and R.H.Teunter, Review of inventory systems with deterioration since 2001, European Journal of Operational Research, 212 (2012) 275-284.

3. J.T.Hso and L.F.Hso, Two EPQ models with imperfect production processes, inspection errors, planned backorders, and sales returns, Computers \& Industrial Engineering, 64 (2013) 389-402.

4. A.Kundu, P.Guchhait, G.Panigrahi and M.Maiti, An imperfect EPQ model for deteriorating items with promotional effort dependent demand, Journal of Intelligent \& Fuzzy Systems, 33 (2017) 649-666.

5. P.K.Ghosh, Optimal imperfect production inventory model under joint effect of machine breakdowns and system reliability, Annals of Pure and Applied Mathematics, 14(2) (2017) 277-291.

6. P.V.Beek and C.V.Puttin, OR contributions to exibility improvement in production /inventory systems, European Journal of Operations Research, 31 (1987) 52-60.

7. E.L.Porteous, Optimal lot sizing, process quality improvement and set-up cost reduction, Operation Research, 34 (1986) 137-144.

8. M.J.Rosenblatt and H.L.Lee, Economic production cycles with imperfect production process, IEEE Transactions, 14 (1986) 48-55.

9. M.Maragatham1 and G.Gnanvel, A purchasing inventory model for breakable items withpermissible delay in payments and price discount, Annals of Pure and Applied Mathematics, 15 (2) (2017) 305-314.

10. P.Guchhait, M.K.Maiti and M.Maiti, Production-inventory models for a damageable item with variable demands and inventory costs in an imperfect production process, Int. J. Production Economics, 144 (2013) 180-188.

11. M.Mandal, and M.Maiti, Inventory of damagable items with variable replenishment and stock dependent demand, Asia Pacific Journal of Operational Research, 17 (2000) 41-54.

12. M.K.Maiti and M.Maiti, Production policy for damageable items with variable cost function in an imperfect production process via genetic algorithm, Mathematical and Computer Modelling, 42 (2005) 977-990.

13. N.H.Shah, and L.E.Cardenas-Barron, Retailers decision for ordering and credit policies for deteriorating items when a supplier offers order-linked credit period or cash discount, Applied Mathematics and Computation, 259 (2015) 569-578.

14. M.G Arif, An inventory model for deteriorating items with non-linear selling price dependent demand and exponentially partial backlogging shortage, Annals of Pure and Applied Mathematics, 16 (1) (2018) 105-116.

15. C.Gurnani, Economic analysis of inventory systems, International Journal of Production Research, 21 (1983) 261-277.

16. I.Moon and W.Yun, An economic order quantity model with random planning horizon, The Engineering Economist, 39 (1993) 77-86.

17. S.Pal, M.K.Maiti and M.Maiti, An EPQ model with price discounted promotional demand in an imprecise planning horizon via genetic algorithm, Computers \& Industrial Engineering, 57 (2009) 181-187. 
An EPQ model for Breakable Item under Imperfect Production Process

18. C.C.Lee and S.L.Hsu, A two-warehouse production model for deteriorating inventory items with time-dependent demands, European Journal of Operational Research, 194(3) (2009) 700-710.

19. A.Kundu, P.Guchhait, P.Paramanik, M.K.Maiti and M.Maiti, A production inventory model with price discounted fuzzy demand using an interval compared hybrid algorithm, Swarm and Evolutionary Computation, 34 (2017) 1-17.

20. P.Mariappan, M.Kameswari and M.A.Raj, Inventory model for deteriorating items with no shortages, Annals of Pure and Applied Mathematics, 15(2) (2017) 327-339.

21. M.Khouja, The economic production lot size model under volume exibility, Computer and Operations Research, 22 (1995) 515-525.

22. M.Khouja and A.Mehrez, An economic production lot size model with imperfect quality and variable production rate, Journal of the Operational Research Society, 45(12) (1994) 1405-1417.

23. T.C.E.Cheng, An economic production quantity model with exibility and reliability consideration, European Journal of Operational Research, 39 (1989) 174-179.

24. L.A.Zadeh, Fuzzy Sets, Information and Control, 8 (1965) 338-356.

25. M.K.Maiti, A fuzzy genetic algorithm with varying population size to solve an inventory model with credit-linked promotional demand in an imprecise planning horizon, European Journal of Operational Research, 13 (2011) 96-106.

26. P.Guchhait, M.K.Maiti and M.Maiti, Two storage inventory model of a deteriorating item with variable demand under partial credit period, Applied Soft Computing, 13 (2013) 428-448.

27. P.Guchhait, M.K.Maiti and M.Maiti, Inventory policy of a deteriorating item with variable demand under trade credit period, Computers \& Industrial Engineering, 76 (2014) 75-88.

28. P.Guchhait, M.K.Maiti and M.Maiti, An EOQ model of deteriorating item in imprecise environment with dynamic deterioration and credit linked demand, Applied Mathematical Modelling, 39 (2015) 6553-6567.

29. S.S.Rao, Engineering Optimization, Theory and Practice, New age International (P) Limited, India, 2000, 715-761.

30. A.Charnes and W.W.Cooper, Chance constrained programming, Management Science, 6 (1959) 73-79.

31. S.H.Chen and C.H.Hsieh, Optimization of fuzzy back order inventory mosdels, IEEE International Fuzzy System Conference (Seoul, Koria) Proceedings, 1 (1999) 240244.

32. S.H.Chen and C.H Hsieh, Representation, ranking, distance, and similarity of L-R type fuzzy number and application, Australian Journal of Intelligent Proceedings Systems, 6 (2000) 217-229. 


\section{A.Kundu, P.Guchhait G.Panigrahi and M.Maiti}

\section{Appendix-A}

A.1. Optimization with stochastic constraints (Rao [29]): Let $x=\left(x_{1}, x_{2}, \ldots, x_{n}\right)^{T}$, be the decision vector, $y=\left(y_{1}, y_{2}, \ldots, y_{n}\right)^{T}$ be the vector of nrandomvariables with known mean and standard deviation, where $y_{1}, y_{2}, \ldots, y_{n}$ represents $n$ parameters of the problem, then a singleobjective optimization problem with stochastic constraints, can be stated in standard form as follows:

Find $\quad x=\left(x_{1}, x_{2}, \ldots, x_{n}\right)^{T}$
which Minimize/Maximize

where $P\left(\phi_{r}(x, y) \geq 0\right)$ represents probability of the event $\left(\phi_{r}(x, y) \geq 0\right)$.

According to Charnes and Cooper [30], if all $y_{i}(i=1,2, \ldots, n)$ follow independent normal distribution, the stochastic problem stated above is equivalent to following crisp nonlinear programming problem

$$
\begin{gathered}
\text { Find } x=\left(x_{1}, x_{2}, \ldots, x_{n}\right)^{T} \\
\text { which } \frac{\text { Minimize }}{\text { Maximize }} \bar{\psi} \\
\text { subject to } \\
\overline{\phi_{r}}-\epsilon_{r}\left[\sum_{i=1}^{N}\left(\frac{\partial \phi_{r}(x, \bar{y})}{\partial y_{i}}\right)^{2} \sigma_{y_{i}}^{2}\right]^{\frac{1}{2}} \geq 0 \quad(r=1,2, \ldots m)
\end{gathered}
$$

where $\overline{\phi_{r}}$ and $\sigma_{\phi_{r}}$ are mean and standard deviation of $\phi_{r}(x, y)$ respectively and also $\epsilon_{r}$ is given by

$$
p_{r}=\frac{1}{\sqrt{2 \pi}} \int_{-\infty}^{\varepsilon_{r}} e^{-t^{2} / 2} d t
$$

\section{Appendix-B}

B.1. Graded Mean Integration Representation (GMIR) of Fuzzy Number:

Chen and Hsieh [31, 32] introduced GMIR method based on the integral value of graded mean $\alpha$-level of generalized fuzzy number. The graded mean $\alpha$-level value of generalized fuzzy number

$$
\tilde{A}=\left(A_{1}, A_{2}, A_{3}, A_{4}\right) \text { is } \alpha\left[\frac{A_{L}(\alpha)+A_{R}(\alpha)}{2}\right], \alpha \in[0,1] .
$$

Then the GMIR of a general fuzzy number $\tilde{A}$

$$
\mathrm{P}(\widetilde{\mathrm{A}})=\int_{0}^{1} \alpha\left[\frac{\mathrm{A}_{\mathrm{L}}(\alpha)+\mathrm{A}_{\mathrm{R}}(\alpha)}{2}\right] \mathrm{d} \alpha / \int_{0}^{1} \alpha \mathrm{d} \alpha=\frac{1}{6}\left[\mathrm{~A}_{1}+2 \mathrm{~A}_{2}+2 \mathrm{~A}_{3}+\mathrm{A}_{4}\right]
$$

Here equal weightage has been given to the left and right parts of the membership function. The representation given by (22) can be generalized/modified by replacing 
An EPQ model for Breakable Item under Imperfect Production Process

$$
\left[\frac{A_{L}(\alpha)+A_{R}(\alpha)}{2}\right], \alpha \in[0,1] \text { with }\left[k A_{L}(\alpha)+(1-k) A_{R}(\alpha)\right], \alpha \in[0,1],
$$

where the value of $k$ depends on the preference of the decision maker. Therefore, the modified form of Eq. (22) is

$P_{k}(\widetilde{\mathrm{A}})=\frac{\int_{0}^{1} \alpha\left[k A_{L}(\alpha)+(1-k) A_{R}(\alpha)\right] \mathrm{d} \alpha}{\int_{0}^{1} \alpha \mathrm{d} \alpha}=\frac{1}{3}\left[\mathrm{k}\left(\mathrm{A}_{1}+2 \mathrm{~A}_{2}\right)+(1-\mathrm{k})\left(2 \mathrm{~A}_{3}+\mathrm{A}_{4}\right)\right.$

The method is also known as k-preference integration representation. For the present investigation, $k=0.5$. 